Pacific Journal of Mathematics

CONVOLUTION TRANSFORMS WHOSE INVERSION 


\title{
CONVOLUTION TRANSFORMS WHOSE INVERSION FUNCTIONS HAVE COMPLEX ROOTS
}

\author{
JOHN DAUNS AND DAVID V. WIDDER
}

The convolution transform is defined by the equation

$$
f(x)=\int_{-\infty}^{\infty} G(x-t) \varphi(t) d t=(G * \varphi)(x) .
$$

If the kernel $G(t)$ has a bilateral Laplace transform which is the reciprocal of an entire function $E(s)$, then $E(s)$ is called the inversion function of the transform. This terminology is appropriate in view of the fact that the transform (1.1) is inverted, in some sense, by the operator $E(D)$, where $D$ stands for differentiation with respect to $x$ :

$$
E(D) f(x)=\varphi(x) .
$$

It is the purpose of the present paper to prove $(1.2)$ when the roots of $E(s)$ are allowed to be genuinely remote from the real axis.

Formula (1.2) was first proved by Widder [7] in 1947 for a large class of entire functions $E(s)$ and by Hirschman and Widder [3] in 1949 for the whole Laguerre-Pólya class. The latter functions have real roots only, indeed are the uniform limits of polynomials with real roots only, see p. 42 of [5].

In 1951 Hirschman and Widder [4] extended this inversion theory, allowing the roots of $E(s)$ to be complex. However, the roots were asymptotically real in the sense that their arguments clustered to 0 or to $\pi$. At the same time A. O. Garder [2] allowed the approach to the real axis to be slower. We require only that they should occur in pairs symmetric in the origin and in a sector inside the sector $|\tan (\arg s)|<1$. More precisely:

$$
\begin{gathered}
E(s)=\prod_{1}^{\infty}\left(1-\frac{s^{2}}{a_{k}^{2}}\right), \sum_{k=1}^{\infty} a_{k}^{-2}<\infty \\
\left|\arg a_{k}\right| \leqq \frac{\pi}{4}-\eta, \quad 0<\eta<\frac{\pi}{4} .
\end{gathered}
$$

We wish also to call attention to some new asymptotic relations.

Received March 18, 1964. Some of this work is part of the first named author's doctoral dissertation at Harvard University. In the preparation of a part of this paper during the year 1962-1963 the first named author was supported by a Graduate National Science Foundation Fellowship (number 22164), and by a grant from the United States Air Force (grant number AF-AFOSR-393-63) during 1963-1964. 
If

$$
G_{2 n}(t)=\prod_{1}^{n}\left(1-\frac{D^{2}}{a_{k}^{2}}\right) G(t)
$$

we show that

$$
G_{2 n}(t) \sim k\left(t, v_{n}\right)
$$

umiformly for $-\infty<t<\infty$. Here $k(t, v)$ is the fundamental solution of the heat equation,

$$
k(t, v)=(4 \pi v)^{-1 / 2} \exp \left(-t^{2} / 4 v\right),
$$

with $-\infty<t<\infty$, with $\operatorname{Re} v>0$, with the square root one-at-one, and where $v_{n}$ is given by

$$
v_{n}=\sum_{n+1}^{\infty} a_{k}^{-2} .
$$

In order to establish (1.3) we are obliged to make an additional assumption on the distribution of the roots of $E(s)$, see Condition $B$ in $\S 4$.

As a consequence of (1.3) we prove that

$$
\int_{-\infty}^{\infty}\left|G_{2 n}(t)\right| d t \sim\left(\cos ^{2} \varphi_{n}-\sin ^{2} \varphi_{n}\right)^{-1 / 2} \quad(n \rightarrow \infty),
$$

where $\varphi_{n}=(1 / 2) \arg v_{n}^{-1}$. This result tends to indicate that present methods cannot be employed for the inversion of (1.1) if the roots of $E(s)$ lie outside the $45^{\circ}$ sector used above.

Finally we compute explicitly the functions $G_{2 n}(t)$ corresponding to $E(s)=\cos \alpha s$ where $|\arg \alpha|<\pi / 2$. Here all roots lie on a line through the origin. In this case the integral (1.4) tends to infinity with $n$ when $|\arg \alpha| \geqq \pi / 4$. This result indicates clearly that our arguments must fail if the roots of $E(s)$ are not restricted to lie inside the $45^{\circ}$ sector.

2. A first inversion theorem. Let us introduce the following conventions.

Condition A. The sequence $a_{1}, a_{2}, \cdots$ of complex constants satisfies Condition A if

$$
\sum_{1}^{\infty}\left|a_{k}\right|^{-2}<\infty \quad \text { and } \quad\left|\arg a_{k}\right| \leqq \frac{\pi}{4}-\eta
$$

for some $\eta$ in $0<\eta<\pi / 4$. It is assumed that the $a_{k}$ are arranged in an order of nondecreasing real parts with $R e a_{1}>0$, i.e.

$$
0<\operatorname{Re} a_{1} \leqq \text { Re } a_{k} \leqq \text { Re } a_{k+1} \quad(k=1,2, \cdots) .
$$

Definition. The class of entire functions $A$ consists of all entire 
functions $E(s)$ of the form

$$
E(s)=\prod_{1}^{\infty}\left(1-\frac{s^{2}}{a_{k}^{2}}\right)
$$

where the roots $\alpha_{k}$ satisfy condition $A$.

For example, cos $(2+i) s$ belongs to the class $A$.

We now state the main theorem of the present section, a result that will be improved in $\S 3$ by more complicated methods.

Theorem 2.1. If for $-\infty<t<\infty$

1.

$$
G(t)=\frac{1}{2 \pi i} \int_{-i \infty}^{i \infty} \frac{e^{s t}}{E(s)} d s
$$

2. $\varphi(t)$ is bounded on compact sets and

$$
\varphi(t)=O\left(e^{\sigma|t|}\right) \quad\left(|t| \rightarrow \infty, 0<\sigma<\text { Re } a_{1}\right) .
$$

3.

$$
f(x)=\int_{-\infty}^{\infty} G(x-t) \varphi(t) d t,
$$

then

$$
\lim _{n \rightarrow \infty} \prod_{1}^{n}\left(1-\frac{D^{2}}{a_{k}^{2}}\right) f(x)=\varphi(x)
$$

at any point $t=x$ of continuity of $\varphi(t)$.

We shall establish this result by the series of Lemmas 2.2, 2.3, and 2.4.

Consider a fixed function $E(s)$ in the class $A$. Then let $E_{2 n}(s)$ be defined by

$$
E_{2 n}(s)=\prod_{n+1}^{\infty}\left(1-\frac{s^{2}}{a_{k}^{2}}\right) \quad(n=0,1,2, \cdots)
$$

Define $S_{n}$ by

$$
S_{n}=\sum_{n+1}^{\infty}\left|a_{k}\right|^{-2} \quad(n=0,1,2, \cdots) .
$$

Let $G_{2 n}(t)$ and $G(t)$ be defined by

$$
\begin{aligned}
G_{2 n}(t)=\frac{1}{2 \pi i} \int_{-i \infty}^{i \infty} \frac{e^{s t}}{E_{2 n}(s)} d s, \quad G(t)=G_{0}(t) \\
\quad(-\infty<t<\infty ; n=0,1,2, \cdots) .
\end{aligned}
$$

If $P_{2 n}(D)$ is defined as 


$$
P_{2 n}(D)=\prod_{1}^{n}\left(1-\frac{D^{2}}{a_{k}^{2}}\right) \quad(n=0,1,2, \cdots),
$$

then the next lemma will show that the integral (2.3) converges, that

$$
P_{2 n}(D) G(t)=G_{2 n}(t),
$$

and furthermore it will give lower bounds of the function $E_{2 n}(s)$ in terms of both $s$ and $n$. It will become clear later that exactly these lower bounds are the ones needed to obtain the required information about the kernels $G_{2 n}(t)$.

Lemma 2.2. Let the roots $a_{k}=r_{k} e^{i \beta_{k}}, \eta, E_{2 n}(s)$, and $S_{n}$ be as in Condition $A$ and equations (2.1) and (2.2).

A. Let $r e^{i \theta}$ with $r>0$ be any point in the angular sector defined by

$$
|\tan \theta| \geqq \tan \left(\frac{\pi}{2}-\frac{\eta}{2}\right) .
$$

Then

$$
\left|E_{2 n}\left(r e^{i \theta}\right)\right| \geqq 1+r^{2} S_{n} \sin \eta
$$

and also

$$
\left|E_{2 n}\left(r e^{i \theta}\right)\right| \geqq 1+r^{4} \sin ^{2} \eta \sum_{n<i<j<\infty} r_{i}^{-2} r_{j}^{-2} .
$$

B. Define $K$ to be the constant

$$
K=\frac{1}{2} \sin \frac{\eta}{2} .
$$

Let $n$ be arbitrary, $n=0,1,2, \cdots$, but fixed. Let $r e^{i \theta}$ with $r>0$ be any point in the triangular region defined by the inequalities

$$
|\tan \theta| \leqq \tan \left(\frac{\pi}{2}-\frac{\eta}{2}\right), \quad|r \cos \theta| \leqq K S_{n}^{-1 / 2}
$$

Then

$$
\left|E_{2 n}\left(r e^{i \theta}\right)\right| \geqq \frac{2}{3}
$$

Proof. A typical term of the infinite product $E_{2 n}\left(r e^{i \theta}\right)$ satisfies $\left[1-\boldsymbol{r}^{2} \boldsymbol{r}_{k}^{-2} e^{2 i\left(\theta-\beta_{k}\right)}\right]\left[1-\boldsymbol{r}^{2} \boldsymbol{r}_{k}^{-2} e^{-2 i\left(\theta-\beta_{k}\right)}\right]=1-2 r^{2} r_{k}^{-2} \cos 2\left(\theta-\beta_{k}\right)+r^{4} r_{k}^{-4}$.

Since in case $A$, the argument $\theta$ satisfies either $\pi / 2-\eta / 2 \leqq \theta \leqq \pi / 2+\eta / 2$ or $-\pi / 2-\eta / 2 \leqq \theta \leqq-\pi / 2+\eta / 2$, and since the argument $\beta_{k}$ of any 
root satisfies $-\pi / 4+\eta \leqq-\beta_{k} \leqq \pi / 4-\eta$, it follows that in case $A$ we have $-\cos 2\left(\theta-\beta_{k}\right) \geqq \sin \eta$. Consequently, by multiplying out the infinite product, we obtain

$$
\left|E_{2 n}\left(r e^{i \theta}\right)\right| \geqq \prod_{n+1}^{\infty} 1+r^{2} r_{k}^{-2} \sin \eta>1+r^{2} S_{n} \sin \eta .
$$

Similarly, we also obtain the second inequality in $A$.

For the proof of $B$, take $k>n$ and restrict $r e^{i \theta}=\sigma+i y$ to the angular sector $|y| \leqq|\sigma| \cot \eta / 2$. By using the latter inequality, we see that a typical term of the infinite product $E_{2 n}(\sigma+i y)$ has the lower bound

$$
\left|1-\frac{(\sigma+i y)^{2}}{r_{k}^{2} e^{2 i \beta_{k}}}\right| \geqq 1-\frac{\sigma^{2}+y^{2}}{r_{k}^{2}} \geqq 1-\frac{\sigma^{2}}{r_{k}^{2}}\left(1+\cot ^{2} \frac{\eta}{2}\right) .
$$

This latter lower bound is positive. The inequalities $r_{k}^{2} S_{n}>1$ and $|\sigma| \leqq K S_{n}^{-1 / 2}$ imply that

$$
\frac{\sigma^{2}}{r_{k}^{2}}\left(1+\cot ^{2} \frac{\eta}{2}\right)=\frac{\sigma^{2}}{4^{2} K r_{k}^{2}}<\frac{1}{4} .
$$

By use of the latter and by multiplying out the infinite product we obtain

$$
\left|E_{2 n}(\sigma+i y)\right|>1-\sum_{p=1}^{\infty} 4^{-p} S_{n}^{-p} \sum_{n<k(1)<\cdots<k(p)<\infty} r_{k(1)}^{-2} \cdots r_{k(p)}^{-2},
$$

where the indices $k(1), \cdots, k(p)$ range over the integers.

Use of the inequality

$$
\sum_{n<k(1)<<<k<(p)<\infty} r_{k(1)}^{-2} \cdots r_{k(p)}^{-2}<S_{n}^{p}
$$

leads to

$$
\left|E_{2 n}\left(r e^{i \theta}\right)\right| \geqq \frac{2}{3}
$$

Thus conclusion $B$ has been established.

The next lemma gives some facts about the kernels $G_{2 n}(t)$. Once the lower bound given by part $A$ of last lemma is available, the next lemma can be proved exactly as in the case of real roots $a_{k}$, see [6; p. 265] and [5; p. 108]; we omit the proof.

Lemma 2.3. Let $E_{2 n}(s), G_{2 n}(t)$, and $P_{2 n}(D)$ be defined by (2.1), (2.3) and (2.4). In particular, the roots $a_{k}$ defining $E_{2 n}(s)$ satisfy condition $A$, and consequently

$$
0<\operatorname{Re} a_{k} \leqq \text { Re } a_{k+1} \quad(k=n+1, n+2, \cdots) .
$$


Let $n=0,1,2, \cdots$ be arbitrary.

A. For any $\sigma$ in $|\sigma|<$ Re $a_{n+1}$,

$$
G_{2 n}(t)=P_{2 n}(D) G(t)=\frac{1}{2 \pi i} \int_{\sigma-i \infty}^{\sigma+i \infty} \frac{e^{s t}}{E_{2 n}(s)} d s .
$$

B. Let $a_{n}$ as a zero of $E_{2 n}(s)$ be of multiplicity $\mu+1$. Then there is a polynomial $p(t)$ of degree $\mu$ such that for any $k$ in - Re $a_{n+1}<$ $k<\operatorname{Re} a_{n+1}$ and any integer $\nu=0,1,2, \cdots$ the following holds

$$
\left(\frac{d}{d t}\right)^{\nu} G(t)=\left(\frac{d}{d t}\right)^{\nu}\left[p(t) e^{-|t| a_{n+1}}\right]+O\left(e^{-k|t|}\right), \quad(|t| \rightarrow \infty) .
$$

C. For all $s=\sigma+i \tau$ with $|\sigma|<\operatorname{Re} a_{n+1}$ and $-\infty<\tau<\infty$

$$
\frac{1}{E_{2 n}(s)}=\int_{-\infty}^{\infty} e^{-s t} G_{2 n}(t) d t, \quad \int_{-\infty}^{\infty} G_{2 n}(t) d t=1 .
$$

In the next lemma a sufficiently good upper bound of the kernel $G_{2 n}(t)$ in terms of both $t$ and $n$ is proved in order to have an inversion formula as an immediate consequence.

LEMMA 2.4. Let $G_{2 n}(t)$ and $S_{n}$ be as defined by equations (2.3) and (2.2). Then there exist constants $M$ and $K$ independent of both $n$ and $t$ such that

$$
\left|G_{2 n}(t)\right| \leqq M S_{n}^{-1 / 2} \exp \left(-K S_{n}^{-1 / 2}|t|\right) \quad(-\infty<t<\infty, n=0,1,2, \cdots) .
$$

Proof. Use of the fact that $G_{2 n}(t)$ is an even function of $t$ and use of Lemma 2.3 shows that

$$
G_{2 n}(t)=\frac{1}{2 \pi} \int_{-\infty}^{\infty} \frac{e^{-(\sigma+i y) t}}{E_{2 n}(\sigma+i y)} d y
$$

provided $\sigma$ satisfies $0<\sigma<r_{n+1} \cos \beta_{n+1}$ (where $r_{n+1} e^{i \beta_{n+1}}$ is that root of $E_{2 n}(s)$ with smallest positive real part). Let $K$ be as in Lemma 2.2 the constant $K=(1 / 2) \sin (\eta / 2)$. Assume for the rest of the proof that $\sigma$ is restricted to $0<\sigma \leqq K S_{n}^{-1 / 2}$. Then since $\cos \beta_{n+1}>1 / \sqrt{2}$, it follows that

$$
0<\sigma \leqq(1 / 2) \sin (\eta / 2) r_{n+1}<r_{n+1} \cos \beta_{n+1} .
$$

By setting $A=\tan (\pi / 2-\eta / 2)$ and using the lower bounds of Lemma 2.2 we obtain

$$
\left|G_{2 n}(t)\right| \leqq \frac{3 A}{2 \pi} \sigma e^{-\sigma t}+\frac{e^{-\sigma t}}{\pi} \int_{\sigma A}^{\infty} \frac{1}{1+y^{2} S_{n} \sin \eta} d y
$$

Replace the lower limit $\sigma A$ in the last integral by 0 , set $\sigma=K S_{n}^{-1 / 2}$ 
and let $M$ be the constant $M=3 A K / 2 \pi+(1 / 2)(\sin \eta)^{-1 / 2}$. Since $G_{2 n}(t)$ is an even function, the last inequality shows that for all $n$ and $t$, the function $G_{2 n}(t)$ satisfies the conclusion of the theorem

$$
\left|G_{2 n}(t)\right| \leqq M S_{n}^{-1 / 2} \exp \left(-K S_{n}^{-1 / 2}|t|\right) .
$$

REMARK. In the previous lemma the constants $M$ and $K$ are functions of $\eta$ only. As $\eta$ tends to $0, M$ tends to $\infty$ and $K$ tends to 0 , thus making the upper bound of the theorem meaningless as $\eta$ tends to 0 . These are phenomena which are typical of the theory and which we will encounter again.

Now we are in a position to prove Theorem 2.1.

Proof. By letting $M_{0}$ be the constant guaranteed by hypothesis 2 of Theorem 2.1, i.e. for any fixed $x$ and all $t$,

$$
|\varphi(x-t)-\varphi(x)| \leqq M_{0} e^{\sigma|t|},
$$

and by using Lemma 2.3 we find that for any $\delta>0$

$$
\begin{aligned}
\mid P_{2 n}(D)(G * \varphi)(x) & -\varphi(x)\left|\leqq \sup _{|t|<\delta}\right| \varphi(x-t)-\varphi(x)\left|\int_{-\infty}^{\infty}\right| G_{2 n}(t) \mid d t \\
& +M_{0} \int_{\delta<|t|<\infty}\left|G_{2 n}(t)\right| e^{\sigma|t|} d t .
\end{aligned}
$$

Replacement of $\left|G_{2 n}(t)\right|$ by its upper bound given by Lemma 2.4,

$$
\left|G_{2 n}(t)\right| \leqq M S_{n}^{-1 / 2} \exp \left(-K S_{n}^{-1 / 2}|t|\right),
$$

and use of the continuity of $\varphi(t)$ at $t=x$ immediately give the theorem.

3. A second inversion theorem. We now remove the boundedness condition on $\varphi(t)$, assumed in Theorem 2.1, assuming here instead only local integrability. The inversion formula will be valid not only at points of continuity of $\varphi(t)$ but at all points of the Lebesgue set for that function.

THEOREM 3.1. If $G(t)$ and $f(x)$ are defined as in Theorem 2.1 with $\varphi(t) \in L^{1}$ in every finite interval and if

$$
\int_{0}^{t} \varphi(u) d u=O\left(e^{\sigma|t|}\right) \quad\left(|t| \rightarrow \infty, 0<\sigma<\operatorname{Re} a_{1}\right),
$$

then

$$
\lim _{n \rightarrow \infty} \prod_{1}^{n}\left(1-\frac{D^{2}}{a_{k}^{2}}\right) f(x)=\varphi(x)
$$

for all $x$ in the Lebesgue set for $\varphi(t)$. 
We first prove a result about the derivative of $G_{2 n}(t)$.

LEMMA 3.2. Let the roots $a_{k}=r_{k} e^{i \beta_{k}}, \eta, G_{2 n}(t)$, and $S_{n}$ be defined by Condition $A$ and equations (2.3) and (2.2). Then there exist constants $M_{1}, K_{1}, M_{2}$, and $K_{2}$ independent of both $n$ and $t$ such that for all $n=0,1,2, \cdots$ the following holds:

A. If $n$ satisfies $S_{n} \geqq 4 r_{n+1}^{-2}$, then

$$
\left|G_{2 n}^{\prime}(t)\right| \leqq M_{1} S_{n}^{-1} \exp \left(-K_{1} S_{n}^{-1 / 2}|t|\right) \quad(-\infty<t<\infty) .
$$

B. If $n$ satisfies $S_{n}<4 r_{n+1}^{-2}$, then

$$
\left|G_{2 n}^{\prime}(t)\right| \leqq M_{2} r_{n+1}^{2} \exp \left(-K_{2} r_{n+1}|t|\right) \quad(-\infty<t<\infty) .
$$

Proof. First conclusion $A$ will be proved. Let $K$ be the constant

$$
K=\frac{1}{2} \sin \frac{\eta}{2}
$$

Restrict $\sigma$ to $0<\sigma \leqq K S_{n}^{-1 / 2}$. The latter guarantees that $0<\sigma<$ $r_{n+1} \cos \beta_{n+1}$ and hence $G_{2 n}^{\prime}(t)$ is given by

$$
G_{2 n}^{\prime}(t)=-\frac{1}{2 \pi} \int_{-\infty}^{\infty} \frac{e^{-(\sigma+i y) t}(\sigma+i y)}{E_{2 n}(\sigma+i y)} d y .
$$

With $A=\tan (\pi / 2-\eta / 2)$, the above becomes

$$
\begin{aligned}
\left|G_{2 n}^{\prime}(t)\right| \leqq \frac{e^{-\sigma t}}{2 \pi} \int_{-\sigma A}^{\sigma A} \frac{\sigma+|y|}{\left|E_{2 n}(\sigma+i y)\right|} d y \\
+\frac{e^{-\sigma t}}{2 \pi} \int_{\sigma A<|y|<\infty} \frac{\sigma+|y|}{\left|E_{2 n}(\sigma+i y)\right|} d y .
\end{aligned}
$$

The assumption that $S_{n} \geqq 4 r_{n+1}^{-2}$ guarantees that $S_{n}-r_{k}^{-2} \geqq 1 / 2 S_{n}$ for all $k>n$. Hence the second lower bound given by part $A$ of Lemma 2.2 becomes

$$
\begin{aligned}
\left|E_{2 n}(\sigma+i y)\right| & \geqq 1+\frac{1}{2} y^{4} \sin ^{2} \eta \sum_{k=n+1}^{\infty} \frac{1}{r_{k}^{2}}\left(S_{n}-\frac{1}{r_{k}^{2}}\right) \\
& \geqq 1+\left(\frac{1}{2} y^{2} S_{n} \sin \eta\right)^{2} .
\end{aligned}
$$

Use of the last inequality and the estimate of part $B$ of Lemma 2.2 in equation (1) gives

$$
\left|G_{2 n}^{\prime}(t)\right| \leqq \frac{3}{2 \pi} A(1+A) \sigma^{2} e^{-\sigma t}+\frac{e^{-\sigma t}}{\pi} \int_{\sigma A}^{\infty} \frac{\sigma+y}{1+\left(\frac{1}{2} y^{2} S_{n} \sin \eta\right)^{2}} d y
$$


Replace the limit $\sigma A$ by 0 in the last integral; define the constants $c_{1}$ and $c_{2}$ as

$$
c_{1}=\int_{0}^{\infty} \frac{1}{1+u^{4}} d u, \quad c_{2}=\int_{0}^{\infty} \frac{u}{1+u^{4}} d u ;
$$

let $K_{1}$ and $M_{1}$ be the constants

$$
K_{1}=K, \quad M_{1}=\frac{3}{2 \pi} A(1+A) K^{2}+\frac{c_{1} \sqrt{2}}{\pi}(\sin \eta)^{-1 / 2} K+\frac{2 c_{2}}{\pi \sin \eta},
$$

and set $\sigma=K S_{n}^{-1 / 2}$. Then equation (2) gives

$$
\left|G_{2 n}^{\prime}(t)\right| \leqq M_{1} S_{n}^{-1} \exp \left(-K_{1} S_{n}^{-1 / 2}|t|\right)
$$

for all $t$ and all $n$ satisfying $S_{n} \geqq 4 r_{n+1}^{-2}$.

For the proof of part $B, G_{2 n}(t)$ has to be expressed in the form

$$
G_{2 n}(t)=\left(g * G_{2 n+2}\right)(t)
$$

where $g(t)$ is the function

$$
g(t)=\frac{1}{2} a_{n+1} e^{-a_{n+1}|t|} \quad(-\infty<t<\infty) .
$$

Differentiation of (3) under the integral sign and an integration by parts gives

$$
G_{2 n}^{\prime}(t)=-\frac{a_{n+1}^{2}}{2} \int_{-\infty}^{\infty} \frac{u}{|u|} e^{-a_{n+1} \mid u !} G_{2 n+2}(t-u) d u \cdot
$$

By use of the estimate

$$
\left|G_{2 n+2}(t)\right| \leqq M S_{n+1}^{-1 / 2} \exp \left(-K S_{n+1}^{-1 / 2}|t|\right)
$$

of Lemma 2.4, equation (4) becomes

(5) $\left|G_{2 n}^{\prime}(t)\right|$

$$
\leqq \frac{1}{2} M r_{n+1}^{2} \int_{-\infty}^{\infty} \exp \left(-\frac{1}{\sqrt{2}} r_{n+1}|u|\right) S_{n+1}^{-1 / 2} \exp \left(-K S_{n+1}^{-1 / 2}|u-t|\right) d u .
$$

By integrating equation (5) by parts we obtain

$$
\begin{aligned}
& \left|G_{2 n}^{\prime}(t)\right| \leqq M K^{-1} r_{n+1}^{2} \exp \left(-\frac{1}{\sqrt{2}} r_{n+1}|t|\right) \\
& \quad \frac{M}{2 \sqrt{2} K} r_{n+1}^{3} \int_{-\infty}^{\infty} \exp \left(-\frac{1}{\sqrt{2}} r_{n+1}|u|-K S_{n+1}^{-1 / 2}|u-t|\right) d u .
\end{aligned}
$$

If $n$ satisfies $S_{n}<4 r_{n+1}^{-2}$ as in conclusion $B$, then

$$
S_{n+1}-r_{n+1}^{-2}=S_{n}-2 r_{n+1}^{-2}<2 r_{n+1}^{-2} \text { and } S_{n+1}^{-1 / 2}>r_{n+1} / \sqrt{3} \text {. }
$$


Substitution of the latter together with the inequality $|t|-|u| \leqq$ $|u-t|$ in equation (6) gives

$$
\begin{aligned}
& \text { (7) } \quad\left|G_{2 n}^{\prime}(t)\right| \leqq M K^{-1} r_{n+1}^{2} \exp \left(-\frac{1}{\sqrt{2}} r_{n+1}|t|\right) \\
& +\frac{M}{2 \sqrt{2} K} r_{n+1}^{3} \exp \left(-\frac{1}{\sqrt{3}} K r_{n+1}|t|\right) \int_{-\infty}^{\infty} \exp \left(-\frac{\sqrt{3}-K \sqrt{2}}{\sqrt{6}} r_{n+1}|u|\right) d u
\end{aligned}
$$

Let $M_{2}$ and $K_{2}$ be the constants

$$
M_{,}=M K^{-1}+M \sqrt{3} K^{-1}(\sqrt{3}-K \sqrt{2})^{-1}, \quad K_{2}=\frac{1}{\sqrt{3}} K .
$$

Then equation (7) shows that

$$
\left|G_{2 n}^{\prime}(t)\right| \leqq M_{2} r_{n+1}^{2} \exp \left(-K_{2} r_{n+1}|t|\right)
$$

holds for all $t$ and all $n$ satisfying $S_{n}<4 r_{n+1}^{-2}$. Hence conclusion $B$ has been ectablished.

Now we prove Theorem 3.1.

Proof. If $\psi(t)$ is given by

$$
\psi(t)=\int_{0}^{t}[\varphi(x-u)-\varphi(x)] d u \quad(-\infty<t<\infty),
$$

then by the hypotheses of Theorem 3.1 there is a constant $M_{0}$ for which

$$
|\psi(t)|<M_{0} e^{\sigma|t|} \quad(-\infty<t<\infty) .
$$

If $t=x$ is in the Lebesgue set of $\varphi(t)$ then for any $\varepsilon>0$ there is a $\delta>0$ such that $|\psi(t)| \leqq \varepsilon|t|$ for any $t$ in $|t| \leqq \delta$. An integration by parts, easily justified by Lemma 2.3 , yields

$$
\left|P_{2 n}(D)(G * \varphi)(x)-\varphi(x)\right| \leqq \varepsilon \int_{-\infty}^{\infty}\left|t G_{2 n}^{\prime}(t)\right| d t+M_{0} \int_{\delta<|t|<\infty}\left|G_{2 n}^{\prime}(t)\right| e^{\sigma|t|} d t .
$$

Replacing $\left|G_{2 n}^{\prime}(t)\right|$ by either one of the two upper bounds given by the last Lemma 3.2 , we easily obtain the conclusion of the theorem.

4. Asymptotic estimates. For the estimates of the present section we need to place further restrictions on the roots of the inversion function.

Condition B. The sequence of complex constants $a_{1}, a_{2}, \cdots$ satisfies Condition $\mathrm{A}$ and in addition

$$
\lim _{n \rightarrow \infty}\left|a_{n}\right|^{4 / 3} \sum_{n}^{\infty}\left|a_{k}\right|^{-2}=\infty .
$$


For example the sequence $a_{n}=n$ satisfies Condition $\mathrm{B}$. The sequence $a_{n}=2^{n}$ satisfies Condition A but not Condition B. In the latter case the above limit becomes

$$
\lim _{n \rightarrow \infty} 2^{4 n / 3}\left(\frac{4}{3 \cdot 2^{2 n}}\right)=0 .
$$

Definition. The entire function $E(s)$ belongs to the class of functions $B$ if

$$
E(s)=\prod_{1}^{\infty}\left(1-\frac{s^{2}}{a_{k}^{2}}\right)
$$

where the roots of $E(s)$ satisfy Condition B.

We can now state the principal result of this section. To do so we adopt the notation of $\S 1$ for the function $k(t, v)$. Set

$$
S_{n}=\sum_{n+1}^{\infty}\left|a_{k}\right|^{-2}
$$

and

$$
v_{n}=\sum_{n+1}^{\infty} \alpha_{k}^{-2}
$$

THEOREM 4.1. If

$$
\begin{array}{rlr}
G(t) & =\frac{1}{2 \pi i} \int_{-i \infty}^{i \infty} \frac{e^{s t}}{E(s)} d s & (E(s) \in B), \\
G_{2 n}(t) & =\prod_{1}^{n}\left(1-\frac{D^{2}}{a_{k}^{2}}\right) G(t) &
\end{array}
$$

then

$$
G_{2 n}(t)=k\left(t, v_{n}\right)+O\left(\left|a_{n+1}\right|^{-2} S_{n}^{-3 / 2}\right) \quad(n \rightarrow \infty)
$$

uniformly on $-\infty<t<\infty$.

Observe that the remainder term in (4.3) tends to zero with $v_{n}$ under the assumption $E(s) \in B$.

Lemma 4.2. Let $E_{2 n}(s), v_{n}$ and $S_{n}$ be defined by (2.1), (4.2) and (4.1) with the roots $a_{k}=r_{k} e^{i \beta_{k}}$ satisfying Condition $B$. Then there exist two strictly positive constants $c$ and $\delta$ such that for any $u$ in $-\delta \leqq u \leqq \delta$ we have

$$
\frac{1}{E_{2 n}\left(i r_{n+1} u\right)}=\exp \left(-r_{n+1}^{2} v_{n} u^{2}\right)+O\left[r_{n+1}^{2} S_{n} u^{4} \exp \left(-c r_{n+1}^{2} S_{n} u^{2}\right)\right] .
$$

The O-term denotes a function of both $n$ and $u$ such that for some constant $M$ and all $u$ and $n$ the absolute value of this function does 
not exceed $M$ times the quantity inside the O-symbol.

Proof. Let $J_{n}(u)$ be the function

$$
J_{n}(u)=\frac{1}{E_{2 n}\left(i r_{n+1} u\right)}-\exp \left(-r_{n+1}^{2} v_{n} u^{2}\right) \quad(n=0,1,2, \cdots) .
$$

Let $\delta$ be arbitrary in $0<\delta<1 / 2$ and assume that $u$ is restricted to $|u| \leqq \delta$ throughout the proof. If $c_{2 p}(n)$ is defined as

$$
c_{2 p}(n)=(-1)^{p}(1 / p) r_{n+1}^{2 p} \sum_{k=n+1}^{\infty} a_{k}^{-2 p} \quad(n=0,1,2, \cdots ; p=1,2, \cdots),
$$

then

$$
J_{n}(u)=\exp \left(-r_{n+1}^{2} v_{n} u^{2}\right)\left\{\exp \left[\sum_{p=2}^{\infty} c_{2 p}(n) u^{2 p}\right]-1\right\} \quad(|u| \leqq \delta) .
$$

It is interesting to observe that $\lim _{n \rightarrow \infty}\left|c_{2 p}(n)\right|=\infty$ for all $p$, if $r_{k}=k^{\alpha}$ with $\alpha$ in $1 / 2<\alpha<3 / 2$. Next it is shown that $c_{2 p}(n)$ satisfies the inequality

$$
\left|c_{2 p}(n)\right| \leqq \frac{1}{p} r_{n+1}^{2} S_{n} \quad(n=0,1,2, \cdots ; p=1,2, \cdots) .
$$

If $N(t)$ and $\theta(t)$ are the functions

$$
N(t)=\sum_{r_{b}<t} 1, \theta(t)=\int_{t}^{\infty} \lambda^{-2} d N(\lambda) \quad(0 \leqq t<\infty),
$$

then $\left|c_{2 p}(n)\right|$ is given by

$$
\left|c_{2 p}(n)\right|=-\frac{1}{p} r_{n+1}^{2 p} \int_{r_{n+1}}^{\infty} t^{-2 p+2} d \theta(t) .
$$

An integration by parts gives the required inequality

$$
\left|c_{2 p}(n)\right|=\frac{1}{p} r_{n+1}^{2} S_{n}-(p-1) r_{n+1}^{2 p} \int_{r_{n+1}}^{\infty} t^{-2 p+1} \theta(t) d t \leqq \frac{1}{p} r_{n+1}^{2} S_{n} .
$$

Use of the inequality $R e v_{n} \geqq S_{n} \sin 2 \eta$ and (2) in equation (1) gives

$$
\left|J_{n}(u)\right| \leqq \exp \left(-r_{n+1}^{2} S_{n} u^{2} \sin 2 \eta\right)\left\{\exp \left[\left(1-\delta^{2}\right)^{-1} r_{n+1}^{2} S_{n} u^{4}\right]-1\right\} \text {. }
$$

Choose any $\delta_{1}$ in $0<\delta_{1}<1$ and consider the two cases:

Case 1. $\left(1-\delta^{2}\right)^{-1} r_{n+1}^{2} S_{n} u^{4} \leqq \delta_{1}$,

(4) Case 2. $\left(1-\delta^{2}\right)^{-1} r_{n+1}^{2} S_{n} u^{4}>\delta_{1}$.

In Case 1, an application of two geometric sum estimates to (3) give the conclusion of the lemma, i.e. 


$$
\left|J_{n}(u)\right| \leqq\left(1-\delta^{2}\right)^{-1}\left(1-\delta_{1}\right)^{-1} r_{n+1}^{2} S_{n} u^{4} \exp \left(-u^{2} r_{n+1}^{2} S_{n} \sin 2 \eta\right) .
$$

For the proof in Case 2, the inequality (3) gives

$$
\begin{aligned}
\left|J_{n}(u)\right| & \leqq \exp \left(-u^{2} r_{n+1}^{2} S_{n} \sin 2 \eta\right) \\
& +\exp \left\{r_{n+1}^{2} S_{n} u^{2}\left[\left(1-\delta^{2}\right)^{-1} u^{2}-\sin 2 \eta\right]\right\} .
\end{aligned}
$$

Now choose $\delta$ as $\delta=(1 / 2)(\sin 2 \eta)^{1 / 2}$. Then using the inequality

$$
\left(1-\delta^{2}\right)^{-1} u^{2}-\sin 2 \eta \leqq-(2 / 3) \sin 2 \eta
$$

and by multiplying (6) by (4), we obtain the conclusion of the lemma for Case 2:

$$
\left|J_{n}(u)\right| \leqq 2\left(1-\delta^{2}\right)^{-1} \delta_{1}^{-1} r_{n+1}^{2} S_{n} u^{4} \exp \left[-u^{2} r_{n+1}^{2} S_{n}(2 / 3) \sin 2 \eta\right] .
$$

Thus (5) and (7) together prove the lemma.

Next Theorem 4.1 is proved.

Proof. The change of variable $y=r_{n+1} u$ in the integral

$$
G_{2 n}(t)=\frac{1}{\pi} \int_{0}^{\infty} \frac{\cos y t}{E_{2 n}(i y)} d y
$$

and Lemma 4.2 imply that

$$
\begin{aligned}
G_{2 n}(t)= & \frac{r_{n+1}}{\pi} \int_{0}^{\delta} \cos \left(r_{n+1} t u\right)\left\{\exp \left(-r_{n+1}^{2} v_{n} u^{2}\right)\right. \\
& \left.+O\left[r_{n+1}^{2} S_{n} u^{4} \exp \left(-c r_{n+1}^{2} S_{n} u^{2}\right)\right]\right\} \mathrm{du}+\int_{\delta r_{n+1}}^{\infty} \frac{\cos t y}{E_{2 n}(i y)} d y .
\end{aligned}
$$

The hypothesis that $\lim _{n \rightarrow \infty} r_{n+1}^{4 / 3} S_{n}=\infty$ guarantees that for all $n$ sufficiently large we have $S_{n}-r_{k}^{-2}>(1 / 2) S_{n}$. Hence for all large $n$ the second lower bound of part A of Lemma 2.2 satisfies

$$
\left|E_{2 n}(i y)\right| \geqq 1+\frac{1}{2} y^{4} \sin ^{2} \eta \sum_{n+1}^{\infty} \frac{1}{r_{k}^{2}}\left(S_{n}-\frac{1}{r_{k}^{2}}\right) \geqq 1+\left(\frac{1}{2} y^{2} S_{n} \sin \eta\right)^{2} .
$$

The latter inequality shows that

$$
\int_{\delta r_{n+1}}^{\infty} \frac{1}{\left|E_{2 n}(i y)\right|} d y=O\left(r_{n+1}^{-3} S_{n}^{-2}\right) \quad(n \rightarrow \infty) .
$$

Note that

$$
r_{n+1}^{-3} S_{n}^{-2}=O\left(r_{n+1}^{-2} S_{n}^{-3 / 2}\right)
$$$$
(n \rightarrow \infty) \text {. }
$$

For any $v$ with $R e v>0$, the function $k(t, v)$ has the representation

$$
k(t, v)=\frac{1}{\pi} \int_{0}^{\infty} e^{-v v u^{2}} \cos t u d u \quad(-\infty<t<\infty) .
$$


Use of (2) and (3) in equation (1) together with some elementary power series estimates of the exponential function give the conclusion of the theorem.

We saw in Theorem 2.1 that the essential step in the proof of the inversion formula was to show that

$$
\int_{-\infty}^{\infty}\left|G_{2 n}(t)\right| d t=O(1) \quad(n \rightarrow \infty) .
$$

The next theorem gives a more precise asymptotic formula for the $L^{1}$-norms of the kernels $G_{2 n}(t)$.

If $\eta$ and $v_{n}$ are as in Condition $\mathrm{A}$ and in equation (4.2), let $\varphi_{n}$ be defined by

$$
v_{n}=\left|v_{n}\right| e^{-2 i \varphi_{n}}
$$

with $\left|\varphi_{n}\right| \leqq \pi / 4-\eta$. The latter implies that in the next corollary we have

$$
\left(\cos ^{2} \varphi_{n}-\sin ^{2} \varphi n\right)^{-1 / 2} \leqq(\sin 2 \eta)^{-1 / 2}
$$

CoRollary 4.3. Let $G_{2 n}(t), \varphi_{n}$, and $\eta$ be as in Theorem 4.1, equation (4.4) and Condition $A$ respectively. Then

$$
\int_{-\infty}^{\infty}\left|G_{2 n}(t)\right| d t \sim\left(\cos ^{2} \varphi_{n}-\sin ^{2} \varphi_{n}\right)^{-1 / 2} \quad(n \rightarrow \infty) .
$$

Proof. Our first estimate of $G_{2 n}(t)$ from Lemma 2.4,

$$
\left|G_{2 n}(t)\right|<M S_{n}^{-1 / 2} \exp \left(-K S_{n}^{-1 / 2}|t|\right),
$$

shows that

$$
\int_{-\infty}^{\infty}\left|G_{2 n}(t)\right| d t \sim \int_{-1}^{1}\left|G_{2 n}(t)\right| d t \quad(n \rightarrow \infty) .
$$

An elementary integration shows that

$$
\int_{-\infty}^{\infty}\left|k\left(t,\left|v_{n}\right| e^{-2 i \varphi_{n}}\right)\right| d t=\left(\cos ^{2} \varphi_{n}-\sin ^{2} \varphi_{n}\right)^{-1 / 2},
$$

and that

$$
\lim _{n \rightarrow \infty} \int_{1<|t|<\infty}\left|k\left(t, v_{n}\right)\right| d t=0 .
$$

Finally, our second estimate of $G_{2 n}(t)$ from Theorem (4.1),

$$
G_{2 n}(t)=k\left(t, v_{n}\right)+O\left(\left|a_{n+1}\right|^{-2} S_{n}^{-3 / 2}\right),
$$

together with the assumption B that $\left|a_{n+1}\right|^{-2} S_{n}^{-3 / 2}$ goes to zero with $1 / n$, gives the conclusion of the theorem, 


$$
\int_{-\infty}^{\infty}\left|G_{2 n}(t)\right| d t \sim\left(\cos ^{2} \varphi_{n}-\sin ^{2} \varphi_{n}\right)^{-1 / 2} \quad(n \rightarrow \infty)
$$

REMARKS 1. If the roots $a_{k}$ defining the kernels $G_{2 n}(t)$ are of the form $a_{k}=r_{k} e^{i \beta}$ for some $|\beta|<\pi / 4$, then $\varphi_{n}=\beta$ for all $n$, and the asymptotic formula of the previous corollary becomes infinite as $\beta \rightarrow \pi / 4$. The latter fact suggests that our present methods cannot be used to generalize the inversion theorem 3.5 in order to allow the roots to lie in any angular sector about the real axis exceeding or even equal to forty five degrees.

2. It is an open question whether all the results of this section are valid if the hypothesis that $\lim _{n \rightarrow \infty} r_{n+1}^{2} S_{n}^{3 / 2}=\infty$ is replaced by the weaker assumption that $\lim _{n \rightarrow \infty} r_{n+1}^{2} S_{n}=\infty$.

3. It is also an open question whether under some assumption similar to Condition B the integral

$$
\int_{-\infty}^{\infty}\left|t G_{2 n}^{\prime}(t)\right| d t
$$

is asymptotic to a constant times $\left(\cos ^{2} \varphi_{n}-\sin ^{2} \varphi_{n}\right)^{-3 / 2}$.

5. An explicit example. In this section the sequence of kernels $G_{2 n}(t)$ is explicitly evaluated corresponding to $E(s)=\cos \left(\pi e^{-i \beta} s\right)$ where $\beta$ is some number in $|\beta|<\pi / 4$.

If $E_{2 n}(s)$ is the function

$$
E_{2 n}(s)=\prod_{n+1}^{\infty}\left(1-\frac{s^{2}}{(k-1 / 2)^{2} e^{2 i \beta}}\right) \quad(n=0,1,2, \cdots),
$$

then as in equation (2.3), the kernel $G_{2 n}(t)$ is given by

$$
G_{2 n}(t)=\frac{1}{2 \pi i} \int_{-i \infty}^{i \infty} \frac{e^{s t}}{E_{2 n}(s)} d s \quad(-\infty<t<\infty ; n=0,1,2, \cdots) .
$$

Let $a$ and $w$ be $a=e^{i \beta}$ and $w=e^{a t}$. For $k>n$, the residue of the integrand $e^{s t} / E_{2 n}(s)$ at $s=(k-1 / 2) a$ is

$$
\frac{a}{\pi} w^{(2 k-1) / 2}(-1)^{k} \prod_{\jmath=1}^{n}\left(1-\frac{(k-1 / 2)^{2}}{(j-1 / 2)^{2}}\right)=c w^{k-1}(-1)^{n+k} \frac{(k+n-1) !}{(k-n-1) !},
$$

where $c$ is defined as

$$
c=\frac{a \exp (a t / 2) 2^{4 n} n !^{2}}{\pi(2 n) !^{2}} .
$$

The kernel $G_{2 n}(t)$ is easily seen to be the sum of the residues in the 
right half plane $R e s>0$, i.e.

$$
G_{2 n}(t)=c w^{n}\left(\frac{d}{d w}\right)^{2 n} \frac{w^{2 n}}{1+w} \quad(-\infty<t<\infty ; n=0,1,2, \cdots) .
$$

By use of the Leibnitz rule for differentiation of products, we obtain

$$
G_{2 n}(t)=\frac{2^{2 n-1} n !^{2}}{\pi(2 n) !} a\left[\operatorname{sech} \frac{a t}{2}\right]^{2 n+1} \quad(-\infty<t<\infty ; n=0,1,2, \cdots)
$$

REMARKs 1. Although the above computation is also valid for any $\beta$ with $\pi / 4<|\beta|<\pi / 2$ it can be shown that

$$
\lim _{n \rightarrow \infty} \int_{-\infty}^{\infty}\left|G_{2 n}(t)\right| d t=\infty
$$

and

$$
\lim _{n \rightarrow \infty} \int_{-\infty}^{\infty}\left|t G_{2 n}^{\prime}(t)\right| d t=\infty
$$

for such a $\beta$.

2. Perhaps the inversion Theorem 2.1 remains valid if the roots $a_{k}$ are allowed to lie in an angular sector of exactly forty-five degrees provided the function $\varphi(t)$ is continuous and of bounded variation at the point $t=x$ at which its value is to be recovered. The latter has been shown to be true in [1] for the special kernel $G(t)$ given by (5.1) with $\beta=\pi / 4$.

\section{REFERENCES}

1. J. Dauns, Convolution transforms whose inversion functions have complex roots, $\mathrm{Ph}$. D. thesis 1963, Harvard University, Cambridge, Mass.

2. A. O. Garder, The inversion of a special class of convolution transforms, M. A. thesis 1950, Washington University, St. Louis, Missouri.

3. I. I. Hirschman and D. V. Widder, The inversion of a general class of convolution transforms, Trans. Amer. Math. Soc. 66 (1949).

4. - Convolution transforms with complex kernels, Pacific J. Math. 1 (1951).

5. - The convolution transform, Princeton, 1955,

6. D. V. Widder, The Laplace transform, 2nd ed., Princeton 1946.

7. - Inversion formulas for convolution transforms, Duke J. Math. 4 (1947).

HARVARD UNIVERSITY 


\title{
PACIFIC JOURNAL OF MATHEMATICS
}

\author{
EDITORS
}

\author{
H. Samelson \\ Stanford University \\ Stanford, California \\ R. M. Blumenthal \\ University of Washington \\ Seattle, Washington 98105
}

\author{
J. DugundjI \\ University of Southern California \\ Los Angeles, California 90007 \\ Richard Arens \\ University of California \\ Los Angeles, California 90024
}

\section{ASSOCIATE EDITORS}
E. F. BECKENBACH
B. H. NEUMaNN
F. WOLF
K. YosIDA

\section{SUPPORTING INSTITUTIONS}

\author{
UNIVERSITY OF BRITISH COLUMBIA \\ CALIFORNIA INSTITUTE OF TECHNOLOGY \\ UNIVERSITY OF CALIFORNIA \\ MONTANA STATE UNIVERSITY \\ UNIVERSITY OF NEVADA \\ NEW MEXICO STATE UNIVERSITY \\ OREGON STATE UNIVERSITY \\ UNIVERSITY OF OREGON \\ OSAKA UNIVERSITY \\ UNIVERSITY OF SOUTHERN CALIFORNIA
}

\author{
STANFORD UNIVERSITY \\ UNIVERSITY OF TOKYO \\ UNIVERSITY OF UTAH \\ WASHINGTON STATE UNIVERSITY \\ UNIVERSITY OF WASHINGTON \\ AMERICAN MATHEMATICAL SOCIETY \\ CALIFORNIA RESEARCH CORPORATION \\ SPACE TECHNOLOGY LABORATORIES \\ NAVAL ORDNANCE TEST STATION
}

Mathematical papers intended for publication in the Pacific Journal of Mathematics should by typewritten (double spaced). The first paragraph or two must be capable of being used separately as a synopsis of the entire paper. It should not contain references to the bibliography. Manuscripts may be sent to any one of the four editors. All other communications to the editors should be addressed to the managing editor, Richard Arens, at the University of California, Los Angeles, California 90024.

50 reprints per author of each article are furnished free of charge; additional copies may be obtained at cost in multiples of 50 .

The Pacific Journal of Mathematics is published quarterly, in March, June, September, and December. Effective with Volume 13 the price per volume (4 numbers) is $\$ 18.00$; single issues, $\$ 5.00$. Special price for current issues to individual faculty members of supporting institutions and to individual members of the American Mathematical Society: $\$ 8.00$ per volume; single issues $\$ 2.50$. Back numbers are available.

Subscriptions, orders for back numbers, and changes of address should be sent to Pacific Journal of Mathematics, 103 Highland Boulevard, Berkeley 8, California.

Printed at Kokusai Bunken Insatsusha (International Academic Printing Co., Ltd.), No. 6, 2-chome, Fujimi-cho, Chiyoda-ku, Tokyo, Japan.

PUBLISHED BY PACIFIC JOURNAL OF MATHEMATICS, A NON-PROFIT CORPORATION

The Supporting Institutions listed above contribute to the cost of publication of this Journal, but they are not owners or publishers and have no responsibility for its content or policies. 


\section{Pacific Journal of Mathematics}

\section{Vol. 15, No. $2 \quad$ October, 1965}

Patrick Robert Ahern, On the generalized F. and M. Riesz theorem......... 373

A. A. Albert, On exceptional Jordan division algebras ................ 377

J. A. Anderson and G. H. Fullerton, On a class of Cauchy exponential

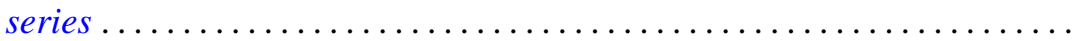

Allan Clark, Hopf algebras over Dedekind domains and torsion in

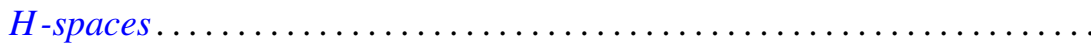

John Dauns and D. V. Widder, Convolution transforms whose inversion functions have complex roots .............................

Ronald George Douglas, Contractive projections on an $\mathrm{L}_{1}$ space ..........

Robert E. Edwards, Changing signs of Fourier coefficients ...............

Ramesh Anand Gangolli, Sample functions of certain differential processes on symmetric spaces .....................................

Robert William Gilmer, Jr., Some containment relations between classes of

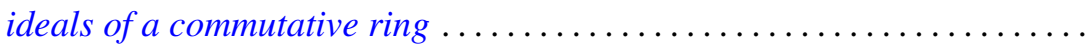

Basil Gordon, A generalization of the coset decomposition of a finite

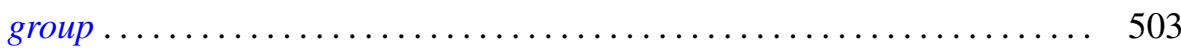

Teruo Ikebe, On the phase-shift formula for the scattering operator....... 511

Makoto Ishida, On algebraic homogeneous spaces ................ 525

Donald William Kahn, Maps which induce the zero map on homotopy ........ 537

Frank James Kosier, Certain algebras of degree one ................. 541

Betty Kvarda, An inequality for the number of elements in a sum of two sets of lattice points.................................

Jonah Mann and Donald J. Newman, The generalized Gibbs phenomenon for regular Hausdorff means. .

Charles Alan McCarthy, The nilpotent part of a spectral operator. II . ...

Donald Steven Passman, Isomorphic groups and group rings ...

R. N. Pederson, Laplace's method for two parameters .....

Tom Stephen Pitcher, A more general property than domination for sets of probability measures .............................

Arthur Argyle Sagle, Remarks on simple extended Lie algebras. .

Arthur Argyle Sagle, On simple extended Lie algebras over fields of

characteristic zero.

Tôru Saitô, Proper ordered inverse semigroups ...........

Oved Shisha, Monotone approximation

Indranand Sinha, Reduction of sets of matrices to a triangular form

Raymond Earl Smithson, Some general properties of multi-valued

functions .................................

John Stuelpnagel, Euclidean fiberings of solvmanifolds .... 